\title{
DETERMINATION OF THE OPTICAL PROPERTIES OF NATIVE JOINT CARTILAGE WITH NIR - SPECTROSCOPY
}

\author{
Reuter $\mathrm{T}^{1}$, Karl $\mathrm{S}^{1}$, Hoffmann $\mathrm{M}^{1}$, Dietzek B ${ }^{2}$, Popp J ${ }^{2}$ \\ ${ }^{1}$ fzmb GmbH, 99947 Bad Langensalza, Germany \\ ${ }^{2}$ IPHT, 07745 Jena, Germany \\ treuter@fzmb.de
}

\begin{abstract}
Current research work at the fzmb deals with the tissue optics and spectral properties of biological tissue. To determine the optical properties of biological tissue, a measurement setup was established, which conforms to the tissue properties absorption, scattering and anisotropy as well as to the underlying requirements of the calculation models. As an example, in this work the optical properties of native joint cartilage are measured.
\end{abstract}

Keywords: Optical Properties, Cartilage, Kubelka-Munk Theory, Ulbricht Sphere, Near Infrared

\section{Introduction}

Nowadays the spectroscopic investigation of biological tissue is an important part of medical research. A branch of these research projects deals with the relation between illnesses and changes in the spectra to be able to recognize illnesses as early as possible. Therefore, the correlation between changes in the near infrared spectra and arthrosis of the knee is investigated at the Research Center for Medicine Technology and Biotechnology. In the course of these investigations, a diagnosis-supporting system was developed to assess knee joint arthrosis. Core of this system is an arthroscopy probe based on reflection spectroscopic principles. The quality assessment of the tissue poses a great challenge in respect of its special optical properties. The strong scattering properties of cartilage tissue make a simple analysis impossible. The theories, which describe the light propagation in very highly scattering media, are the radiation transport theory and the Kubelka-Munk theory. Within the scope of this work a measurement setup is established to investigate the optical properties of biological tissue.

\section{Methods}

Here, the experimental setup and the KubelkaMunk theory for the determination of the optical properties of biological tissue are described.

\section{Experimental Setup}

For the determination of the optical properties in accordance to Kubelka-Munk, three independent measurements must be carried out: diffuse reflection, diffuse transmission and collimate transmission. For the diffuse reflection and transmission, Ulbricht's spheres were used for the lighting and the detection [1]. A white standard with a reflection rate of $\mathrm{m}=99 \%$ served as a reference. A component, which all used measurement setups have in common, is the spectrometer system "MCS NIR" by Zeiss®.

The cartilage samples were obtained from the knee joint of a pig. The specimens are from the femoral condyle of the joint cartilage and from tissue of the tibial plateau.

\section{Kubelka-Munk Theory}

The basic idea for the description of the propagation of light in a scattering medium is the division of the beam path within the medium in two directions, one in propagation direction of the irradiated light and another in the opposite direction [2]. Furthermore the light propagation in the medium has to be isotropic and diffuse [1][3]. The light should spread in an infinitely expanded disc in z-direction. For a homogeneous diffuse lighting, a distribution of the light therefore arises in the direction of i. Analogously, this is also valid for the back-scattered light in the direction of $\mathrm{j}$. Loss of light during the passage through a layer dz originate from scattering, described by the scattering cross section $\sigma$, and from absorption, defined by the absorption cross section $\alpha$. From the equations of the attenuation of light by a layer $\mathrm{dz}$ in the direction of $\mathrm{i}$ and $\mathrm{j}$ the following coupled differential equation system can be established (see Eq. 1).

$$
\begin{aligned}
& -d i=-(K+S) i d z+S j d z \\
& +d j=-(K+S) j d z+S i d z
\end{aligned}
$$

Derived from these two equations are the conditional equations for the Kubelka-Munk parameters $\mathrm{S}$ and $\mathrm{K}$, which were determined by the measurement of diffuse reflection $R_{d}$ and diffuse transmission $T_{d}$. To describe the relation between the dimensions of the Kubelka- Munk theory and to those of the radiation transport equation, the transformations of Star were used [1][4]. Now, with the help of these equations, the absorption, scattering and anisotropy are combined with the Kubelka-Munk parameters in order to calculate the optical properties from the experimental data.

\section{Results}

The optical properties of cartilage from the femoral condyle and the tibial plateau are depicted in the Figure 1, 2 , and 3. 


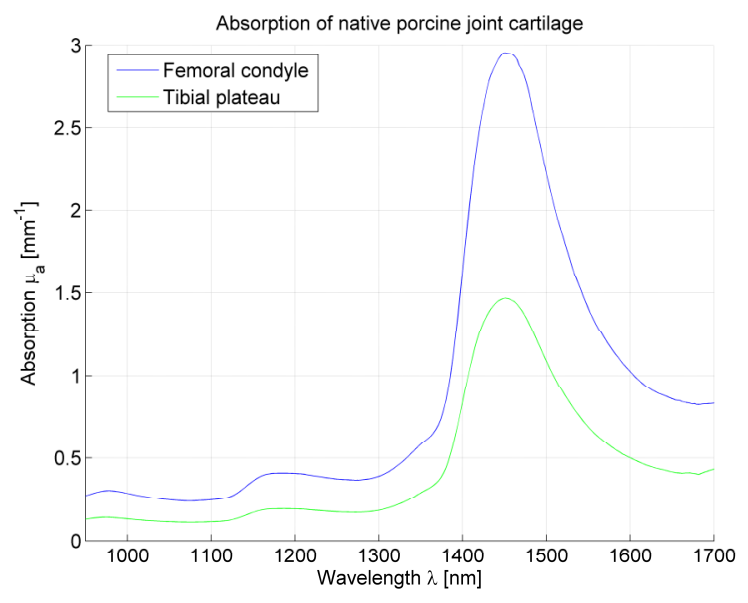

Figure 1: Absorption of native joint cartilage

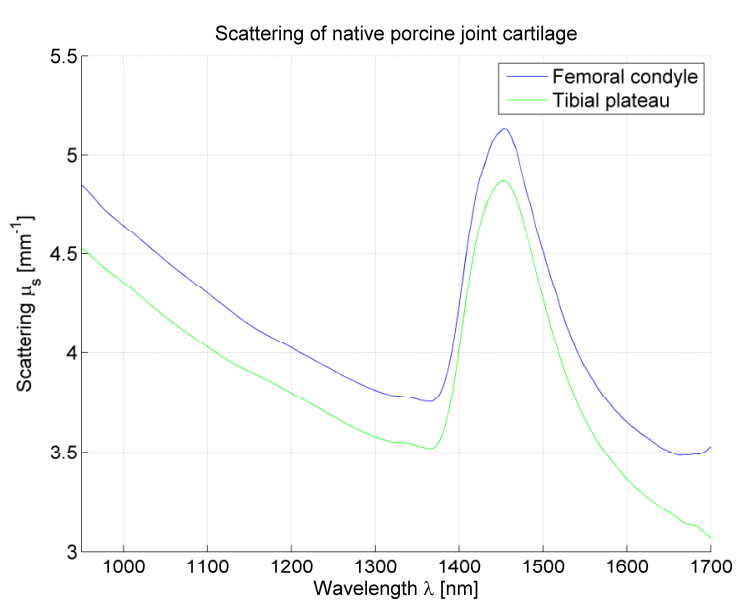

Figure 2: Scattering of native joint cartilage

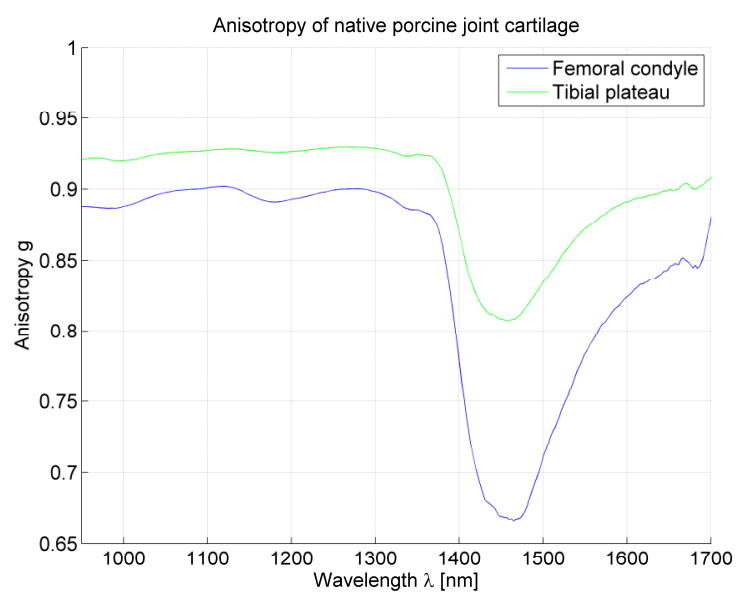

Figure 3: Anisotropy of native joint cartilage

It is recognizable that spectra of absorption, scattering and anisotropy of the samples are alike in the trend. The values of the absorption rise in particular in the area of the water absorption at about $1,440 \mathrm{~nm}$ almost to the level of the scattering. Additionally, the cartilage of the femoral condyle exhibits a higher absorption in the water area, than that of the tibial plateau. Nevertheless, the scattering represents the dominant mechanism of the attenuation of light beyond the water area. Furthermore, the cartilage shows a distinctive forward scattering with a anisotropy of about 0.9 . Only in the area of the water absorption it drops to 0.8 and 0.65 for the tibial plateau and the femoral condyle, respectively.

\section{Discussion}

At the fzmb a setup could be realized to measure the optical properties of very highly scattering media. The measurement of the optical properties of cartilage samples from the femoral condyle and the tibial plateau exhibited a strong absorption peak at $1,440 \mathrm{~nm}$. The scattering and the anisotropy of both samples differ only slightly. Beyond the water peak the scattering is also the main mechanism for the attenuation of light in the cartilage due to about ten times higher values. In the area of the water absorption it is only about twice as high. Further research is concerned with the development of models close to reality of the tissue optics, serving as a basis for the development and optimization of diagnostic and therapeutic systems.

\section{Acknowledgement}

This study is part of a research project supported by the German Ministry of Economy and Technology (VF090058). The authors would like to thank Petra Prokop and Nastja Rebrin for translation and editing.

\section{Bibliography}

[1] Cheong WF, Prahl SA, Welch AJ: A Review of the Optical Properties of Biological Tissues, Quantum Electronics, IEEE Journal of, vol.26, no.12, pp.2166-2185, 1990.

[2] Splinter R, Hooper BA: An Introduction To Biomedical Optics, CRC Press, Taylor \& Francis Group, 2007.

[3] Molenaar R, Bosch JJ, Zijp JR: Determination of Kubelka-Munk Scattering and Absorption Coefficients by Diffuse Illumination, Appl. Opt. 38, pp. 2068-2077, 1999.

[4] Thennadil SN: Relationship between the Kubelka-Munk scattering and radiative transfer coefficients, J. Opt. Soc. Am. A 25, pp. 1480-1485, 2008. 